\title{
Un modelo pedagógico virtual centrado en las E-actividades
}

\section{A virtual pedagogical model centered on E-activities}

\author{
Juan Silva \\ Universidad de Santiago de Chile. Santiago de Chile. Chile \\ juan.silva@usach.cl
}

\begin{abstract}
Resumen
El uso de los Learning Management Systems (LMS), ha sido incorporado crecientemente en la docencia para complementar la clase presencial o introducir modalidades de elearning. Sin embargo, se observa un pobre uso de los LMS para innovar en las propuestas pedagógicas. Un pertinente y estudiado uso de las metodologías de aprendizaje en red, podría contribuir en el uso de los LMS para el diseño de entornos virtuales de aprendizaje (EVA) innovadores, que den vida a modelos pedagógicos que favorezcan el aprender haciendo, dando cabida a diferentes estilos de aprendizaje y generar en los estudiantes competencias asociados al aprendizaje autónomo, en red y colaborativo. Se requiere un cambio metodológico que permita transitar de un método centrado en el contenido y el profesor, a uno centrado en las e-actividades y el alumno. Este artículo presenta una propuesta de un modelo pedagógico online centrado en las e-actividades. Un espacio para la construcción de conocimiento, al interior de una comunidad de aprendizaje, basada en el diseño instruccional online, donde las actividades son el centro, articulando la colaboración, la tutoría, los recursos, contenidos y la plataforma. El modelo es flexible se adapta a diferentes contextos, temáticas y tipos de estudiantes.
\end{abstract}

\section{Palabras clave}

e-actividades, entorno virtual de aprendizaje, e-learning, metodologías, aprendizaje centrado en el alumno.

\begin{abstract}
The use of Learning Management Systems ( LMS ), have increasingly been incorporated in teaching to supplement class time or introduce e-learning modalities . However a poor use of LMS is noted for innovation in the educational proposals. A relevant and studied use of networked learning methodologies , could contribute to the use of LMS for designing virtual learning environments (EVA ) innovative, that give life to pedagogical models privileging virtual online learning by doing, allowing for different learning styles and build on students skills associated with independent learning, networking and collaborative. It requires a methodological change that allows to move from a content-centric and teachercentered approach to one focused on e-activities and the learner. This article presents a proposal for a pedagogical model focused on online e-activities. A space for the construction of knowledge, within a learning into community, based on the online instructional design, where activities are central, coordinating collaboration, mentoring, resources, content and platform. The model is flexible and adapting in different contexts, themes and types of students.
\end{abstract}

\section{Key words}

e- activities, virtual learning environment, e-learning methodologies, studentcentered learning Social networking, collaborative work, higher education, learning theories, gender. 


\section{Introducción}

Los Learning Management Systems (LMS), permiten el diseño de entornos virtuales de aprendizajes (EVA), espacios formativos que para innovar en la docencia requieren utilizar metodologías centradas en el alumno, cambio de roles en docentes y estudiantes, incorporar las TIC para colaborar, compartir y distribuir conocimiento en red. Especialmente a nivel universitario, se ha masificado el uso de LMS integrado como apoyo a la docencia en modalidades: apoyo a la clase presencial, b-learning o e-learning (García Aretio, Ruiz \& Domínguez, 2007; Barbera \& Badia, 2004).

El aula virtual es considerada una innovación en la inserción de las TIC en la docencia Universitaria. Sin embargo, la experiencia y la literatura demuestran que normalmente se transfiere al espacio virtual un modelo tradicional de formación, que apelan a metodologías conductistas. Se pasa del aula presencial al aula virtual, recreando los mismos espacios y funcionamientos (Adell, 2004). Se requiere avanzar en el diseño de EVA, centrados en el estudiante, de modo de generar experiencias de formación virtual de calidad que aporten a un aprendizaje significativo de los estudiantes (Silva, 2011). Es necesario que los docentes adquieran las competencias necesarias para la integración de las TIC en su práctica docente a nivel general y específicamente en el área virtual diseñando y moderando EVA, migrando desde las metodologías centradas en el profesor, hacia metodologías y e-actividades centradas en el estudiante (Miranda, Guerra, Fabbri \& López, 2010). Este artículo presenta la propuesta de un modelo pedagógico de formación online centrado en las e-actividades, se presenta las bases conceptuales y su implementación en un LMS. El uso de las tecnologías de la información y comunicación (TIC) nos entregan la oportunidad de innovar y renovar las propuestas metodológicas. Se requiere favorecer el uso de metodologías innovadoras para la incorporación de las TIC en los procesos educativos (Gisbert \& Johnson, 2015).

\section{Los nuevos aprendices}

Estamos en presencia de una generación de jóvenes, la cual está fuertemente identificada y familiarizada con el uso de las tecnologías, se han formado y han crecido en la era digital. Esta generación se caracteriza por superar a sus profesores/adultos en el dominio de las tecnologías y tienen más fácil acceso a datos, información y conocimientos que circulan en la red y por vivir en una cultura de interacción y su paradigma comunicacional se basa en la interactividad al usar un medio instantáneo y personalizable como Internet (Oblinger \& Oblinger, 2005). Esta generación de estudiantes vive la tecnología como parte de su entorno habitual, para ellos la información y el aprendizaje no están relegados a los muros del aula, ni es ofrecida en forma exclusiva por el profesor.

Un estudio sobre el uso de la Internet en las universidades Catalanas, llegó a la conclusión que los estudiantes que acceden a un uso intensivo y creativo de las herramientas de la Web especialmente herramientas web 2.0, suelen tener más 
problemas para adaptarse a la enseñanza tradicional que la universidad les ofrece, incluso tener más bajo rendimiento (Duart et al. ,2008). Esto último no se asocia con falta de capacidades, sino porque esperarían educarse en ambientes más creativos que utilizan las herramientas TIC empleadas normalmente en sus vidas. Los estudios en estudiantes universitarios en el aprendizaje informal, muestran que los ambientes de aprendizaje formales desconocen y desaprovechan la forma en que los estudiantes utilizan las TIC para aprenden fuera de las aulas universitarias (Carrasco, Jadue, Letelier \& Oliva, 2012). Se requiere un uso innovador de las TIC para convertir al estudiante en un actor protagónico de su aprendizaje, permitiéndole opinar, interactuar y aportar conocimiento a la red (Pedró, 2006).

La literatura muestra que no hay una generación digital homogénea, se presentan diferentes perfiles derivados del acceso a los aparatos tecnológicos, las horas de exposición frente a ellos y los tipos de usos (Kennedy et al,, 2008). Presentan un mejor manejo de las competencias tecnológicas asociadas a actividades sociales y lúdicas, pero siguen sin adquirir las competencias que les permitan dominar esas herramientas en el ámbito educativo y en su desarrollo profesional, impactando directamente en sus procesos de aprendizaje y construcción de conocimiento (Esteve, Duch \& Gisbert, 2014). Las autoras Escofet, García y Gros (2012) ponen énfasis en la relación "usos informales" y "usos formales", diferenciando tecnologías y usos de éstas para vivir y para aprender. Su conclusión es que los jóvenes usan las TIC para vivir, pero no para aprender o desenvolverse en las labores prácticas del trabajo formativo.

Un estudio con estudiantes universitarios españoles y chilenos, muestra que éstos se encuentran preparados para participar con éxito en procesos formativos en modalidad virtual (Roig, Rioseco \& Belmar, 2015). De acuerdo al estudio los estudiantes presentan cuatro competencias: las competencias instrumentales TIC (de ofimática, entornos web, sistemas operativos, etc.); las competencias instrumentales generales (comprensión lectora, escritura, síntesis, acceso a la información, comunicar ideas, etc.); las competencias interpersonales (habilidades para entablar relaciones interpersonales, capacidad para trabajar en equipo, disposición para compartir información, etc.); las competencias sistémicas (condiciones para el aprendizaje, liderazgo, adaptación a nuevas situaciones, a otras culturas y contextos, habilidades para investigar, etc.).

Existen diferentes aproximaciones al tema de las nuevas generaciones y su relación con el mundo digital. Estás pueden ordenarse según su énfasis en tres categorías: el extendido uso de las TIC; el impacto de la inmersión digital particularmente en el aprendizaje; las características personales y de comportamiento distintivo de esta generación (Bullen \& Morgan, 2015). Las dos últimas categorías tienen particular relevancia en la implementación modalidades 
de aprendizaje a distancia abiertas y flexible, como las que ofrecen las plataformas online, los MOOC, el M-learning, Serious Game, entre otras tecnologías que facilitan el aprendizaje online.

\section{Los Entornos Virtuales de Aprendizaje}

Un entorno virtual de aprendizaje (EVA) es una aplicación informática diseñada para facilitar la comunicación pedagógica entre los participantes en un proceso educativo, sea éste completamente a distancia, presencial, o de una naturaleza mixta que combine ambas modalidades en diversas proporciones, (Adell, Castellet \& Pascual, 2004). En un EVA se encuentran espacios para: distribuir materiales educativos en formato digital (textos, imágenes, audio, simulaciones, juegos, etc.), realizar discusiones en línea, integrar contenidos relevantes de la red o para posibilitar la participación de expertos o profesionales externos en los debates o charlas.

Un EVA es la creación de materiales informáticos de enseñanza-aprendizaje basados en un sistema de comunicación mediada por el computador, lo que se diferencia de una página web (Gros, 2004). Esta autora considera que el diseño de un entorno para la formación debería tener en cuenta una serie de características específicas que proporcionen el medio a partir de las cuales plantear su explotación. Dillenbourg (2000), destaca siete elementos básicos de un EVA: es un espacio diseñado con finalidades formativas; es un espacio social; el espacio social está representado explícitamente; los estudiantes no sólo son activos sino también actores, co-construyen el espacio virtual; no están restringidos a la enseñanza a distancia; integran diferentes tecnologías y también enfoques pedagógicos múltiples; no excluyen necesariamente los entornos físicos.

En un EVA se combinan herramientas para: la comunicación sincrónica y asincrónica; gestión de los materiales de aprendizaje; gestión de las personas participantes; sistemas de seguimiento y evaluación del progreso de los estudiantes; soporte tecnológico a profesores y estudiantes para optimizar distintas fases del proceso de enseñanza/aprendizaje: planificación, implementación, desarrollo y evaluación del currículum (García Areito et al., 2007).

La implementación de un EVA, no garantiza la innovación ni la mejora de la calidad de la enseñanza (Adell, 2004). Diseñar e implementar un EVA para innovar en las prácticas formativas online, requiere planes de formación del profesorado que consideren: la competencia digital docentes (Gisbert \& Lazaro, 2015); metodologías para la enseñanza aprendizaje centrado en el estudiante apoyadas por TIC; el rol del profesor como diseñador y moderador de EVA (Silva, 2011). 


\section{Metodologías centradas en el estudiante}

Por metodologías activas se entiende aquellos métodos, técnicas y estrategias que utiliza el docente para convertir el proceso de enseñanza en actividades que fomenten la participación activa del estudiante y lleven al aprendizaje (Labrador \& Andreu, 2008). Estas metodológicas activas, redefinen el proceso de enseñanzaaprendizaje, pasando de la enseñanza tradicional centrada en el docente y la clase magistral a un proceso formativo centrado en el alumno, cambiando el foco de los contenidos a las actividades. Transitando de una enseñanza que fomenta alumnos pasivos expuestos a metodologías expositivas, a un aprendizaje centrado en el alumno, que favorezca su actividad y protagonismo. Estas metodologías activas permiten responder de mejor forma a los estilos de aprendizaje que presentan los estudiantes a la hora de enfrentarse a las tareas educativas (Bolívar \& Rojas, 2014).

Los enfoques metodológicos de carácter socio-constructivista e investigador jugarán un papel muy significativo en los EVA, dadas las posibilidades de establecer comunicaciones sincrónicas y asincrónicas entre los diferentes miembros de la comunidad de aprendizaje, ofrecer experiencias contextualizadas y significativas para el alumno (Martín \& López, 2012). Esto implica necesariamente desarrollar ajustes en los objetivos y contenidos de las propuestas de formación eLearning, el replanteamiento de la metodología y la concreción de tareas que pongan atención al proceso por sobre el suceso de evaluación final y los contenidos memorísticos.

La inserción de las TIC en la educación de forma que estás realmente produzcan una innovación en la docencia y contribuyan a producir más y mejores aprendizajes, requiere un cambio metodológico El éxito de la inserción de TIC en la educación va de la mano de cambios metodológico. En este sentido Salinas (2004) señala que durante los primeros años de utilización de las TIC, los proyectos se centraron en la innovación técnica para crear entornos de aprendizaje basados en la tecnología. Ahora el foco es el alumno mismo, así como la metodología. Este nuevo pensamiento demanda una sólida formación metodológica, al mismo tiempo que un enfoque centrado en el alumno.

Con el advenimiento de las TIC no se crean metodologías nuevas, más bien las ya existentes se potencian a partir de las posibilidades que ofrecen las TIC de búsqueda y acceso a información, interacción, colaboración y ampliar la clase más allá de las fronteras del aula. Para Mason (1998), no se inventan nuevas metodologías, sino que la utilización de las TIC abre nuevas perspectivas respecto a una enseñanza mejor, apoyada en entornos en línea cuyas estrategias son prácticas habituales en la enseñanza presencial, pero que ahora son simplemente adaptadas y redescubiertas en su formato virtual. 
Existe una serie de técnicas asociadas al uso de las TIC, a las cuales se asocian metodologías centradas en el estudiante, quien construye su propio conocimiento en una acción didáctica y en el marco de una estrategia de aprendizaje liderada por el docente (Salinas, Pérez \& De Benito, 2008).

\begin{tabular}{lrl}
\hline Técnica & Metodología \\
\hline Técnicas para la & Búsqueda y organización de la información \\
Individualización de & Contratos de Aprendizaje \\
la Enseñanza & Estudio con Materias (presentaciones, artículos online, \\
& blogs etc.) & Ayudante Colaborador \\
Técnicas Expositivas & Exposición Didáctica (conferencias online, videos) \\
y Participación en & Preguntas de Grupo (Foro online o wiki, google drive, \\
Gran Grupo. & etc.) \\
& Simposio o Mesa Redonda \\
& Tutoría online (herramientas de plataforma, mensajería, \\
& chat, videoconferencia, etc) \\
& Exposiciones de los alumnos Presentaciones multimedia, \\
& vídeos, blogs, etc. \\
Trabajo en Parejas \\
Lluvia de Ideas (herramientas para mapas mentales o \\
mapas conceptuales) \\
Simulaciones y juegos de roles \\
Estudio de Casos \\
Aprendizaje Basado en Problemas \\
Investigación social \\
Debate \\
Trabajo por proyectos \\
Grupos de Investigación
\end{tabular}

Tabla 1: Metodologías para el trabajo en red

Estas metodologías favorecen el aprendizaje centrado en las actividades más que en los contenidos, implicando cambios profundos en el actuar de alumnos y docentes, y en la forma en que se planifica el curriculum, se diseñan y planifican las asignaturas y las respectivas clases. Gros (2011) establece diferencias entre concebir el proceso de enseñanza centrado en los contenidos o centrado en las actividades. 


\begin{tabular}{|c|c|c|}
\hline $\begin{array}{l}\text { Aprendizaje centrado } \\
\text { en los contenidos }\end{array}$ & & $\begin{array}{l}\text { Aprendizaje centrado } \\
\text { en las actividades }\end{array}$ \\
\hline $\begin{array}{l}\text { El estudiante suele ser reactivo y pasi- } \\
\text { vo, a la espera de lo que diga o decida } \\
\text { el docente. }\end{array}$ & $>$ & $\begin{array}{l}\text { Los estudiantes tienen una implica- } \\
\text { ción activa en su aprendizaje, sin es- } \\
\text { perar que el docente decida por ellos. }\end{array}$ \\
\hline $\begin{array}{l}\text { El margen de decisión del estudiante } \\
\text { es pequeño. }\end{array}$ & $>$ & $\begin{array}{l}\text { Mucha libertad para los estudiantes y } \\
\text { espacio para las propias decisiones en } \\
\text { cuanto a ciertos elementos importan- } \\
\text { tes de su aprendizaje. }\end{array}$ \\
\hline Se fomenta un aprendizaje individual. & $>$ & $\begin{array}{l}\text { Se fomenta un aprendizaje en colabo- } \\
\text { ración con los compañeros. }\end{array}$ \\
\hline $\begin{array}{l}\text { Los estudiantes no tienen muchas } \\
\text { oportunidades para aprender autóno- } \\
\text { mamente. }\end{array}$ & $>$ & $\begin{array}{l}\text { Los estudiantes tienen ocasiones de } \\
\text { ser autónomos en su aprendizaje. }\end{array}$ \\
\hline $\begin{array}{l}\text { Competencias memorísticas y de re- } \\
\text { plicación de contenidos. }\end{array}$ & $>$ & $\begin{array}{l}\text { Competencias relacionadas con } \\
\text { procesos, con una orientación a re- } \\
\text { sultados, y a la búsqueda, selección y } \\
\text { manejo de información. }\end{array}$ \\
\hline $\begin{array}{l}\text { La educación personal y profesional } \\
\text { a menudo está restringida a periodos } \\
\text { determinados de la vida. }\end{array}$ & $>$ & $\begin{array}{l}\text { Educación personal y profesional a lo } \\
\text { largo de la vida. }\end{array}$ \\
\hline
\end{tabular}

Figura 1: Aprendizaje basado en contenido vs Aprendizaje centrado en actividades Gros, B (2011, Pág. 39).

El aprendizaje centrado en las actividades sitúa al estudiante en el centro del proceso formativo, le entrega un rol protagónico, favorece el aprendizaje colaborativo y autónomo. Estas últimas habilidades de orden superior demandadas por la sociedad del conocimiento y útiles no tan solo para la vida académica sino también para la profesional. Las metodologías activas centran el proceso educativo en el estudiante para generar un aprendizaje situado, en el cual el docente es un mediador orientado hacia el aprendizaje en lugar de la enseñanza, fomentando la participación colaboración, cooperación, la creatividad, la reflexión el análisis y la crítica. Se permite dar respuestas a los estilos de aprendizaje considerados por Honey \& Mumford (2000) como: reflexivo, teórico, pragmático y activo.

\section{Las E-actividades}

Las actividades realizadas a través de entornos virtuales son denominadas por algunos autores como e-actividades (Barberá \& Badia, 2004; Cabero \& Román, 2006; Salmon, 2004). Corresponden a las actividades destinadas al logro de los objetivos, competencias o resultados de aprendizaje, a través de diversas acciones tendientes a facilitar el proceso de enseñanza-aprendizaje. A través de las eactividades los estudiantes ponen en juego habilidades y competencias de diversa índole, que bajo metodologías activas apoyadas por las TIC, posibilitan un aprendizaje significativo, tanto a nivel individual como grupal. La formulación, diseño e implementación de e-actividades son parte central del diseño instruccional online, pueden responder a diferentes finalidades como: la motivación inicial hacia la material; las formativas orientadas a la consecución de objetivos, competencias o resultados de aprendizaje; las evaluativas, que permiten 
constatar el nivel de progreso de los estudiantes (Villalustre \& Del Moral, 2011). Para que las e-actividades favorezcan el aprendizaje autónomo, se requiere que el estudiante asuma un rol activo en su proceso formativo, mediante el planteamiento de parte del docente de tareas que impliquen el desarrollo de estrategias de identificación y gestión del conocimiento (Corno \& Randi, 2000).

El diseño de las e-actividades debe ser cuidadoso para lograr un alto nivel de implicación, motivación e interés hacia la materia, al tiempo de favorecer un aprendizaje autónomo, activo y constructivo (Cabero \& Román, 2006). Entre los principales elementos de calidad en el diseño instruccional online, se encuentra el correcto diseño de la organización interna de las e- actividades (Salinas, Pérez \& De Benito, 2008). Las e-actividades se presentan en un esquema que recoge diversos elementos asociados a la organización de las actividades, como descripción, objetivo, tiempo, acciones, evaluación, producto, entre otros, consideran aspectos orientados tanto a los participantes como a los tutores (Salmon 2004; Salinas et al. 2008; Silva, 2011).

\section{Modelo centrado en las E-actividades}

El modelo se basa en una propuesta metodológica de aprendizaje centrada en el estudiante, que se establece en la convicción de que los sujetos aprenden "haciendo" e "interactuando". Desde este punto de vista, se fomenta el desarrollo de "actividades" que privilegien el trabajo práctico, en equipo y relacionado con situaciones concretas orientadas a la resolución de problemas o el desarrollo de habilidades de orden superior. En la generación de esta propuesta, hemos tomado en cuenta las aportaciones de diversos autores y la experiencia del Centro de Investigación e Innovación en Educación y TIC (CIIET) de la Universidad de Santiago de Chile, que por más de una década ha diseñado e implementado cursos online centrados en las actividades (Silva, 2011; Silva \& Romero, 2014). Se ha aplicado principalmente en contextos de formación continua de docentes en modalidades e-learnig y b-learning, en menor medida en formación de profesionales de otras áreas, actualmente se explora su uso en programas blearning en Educación Superior. 


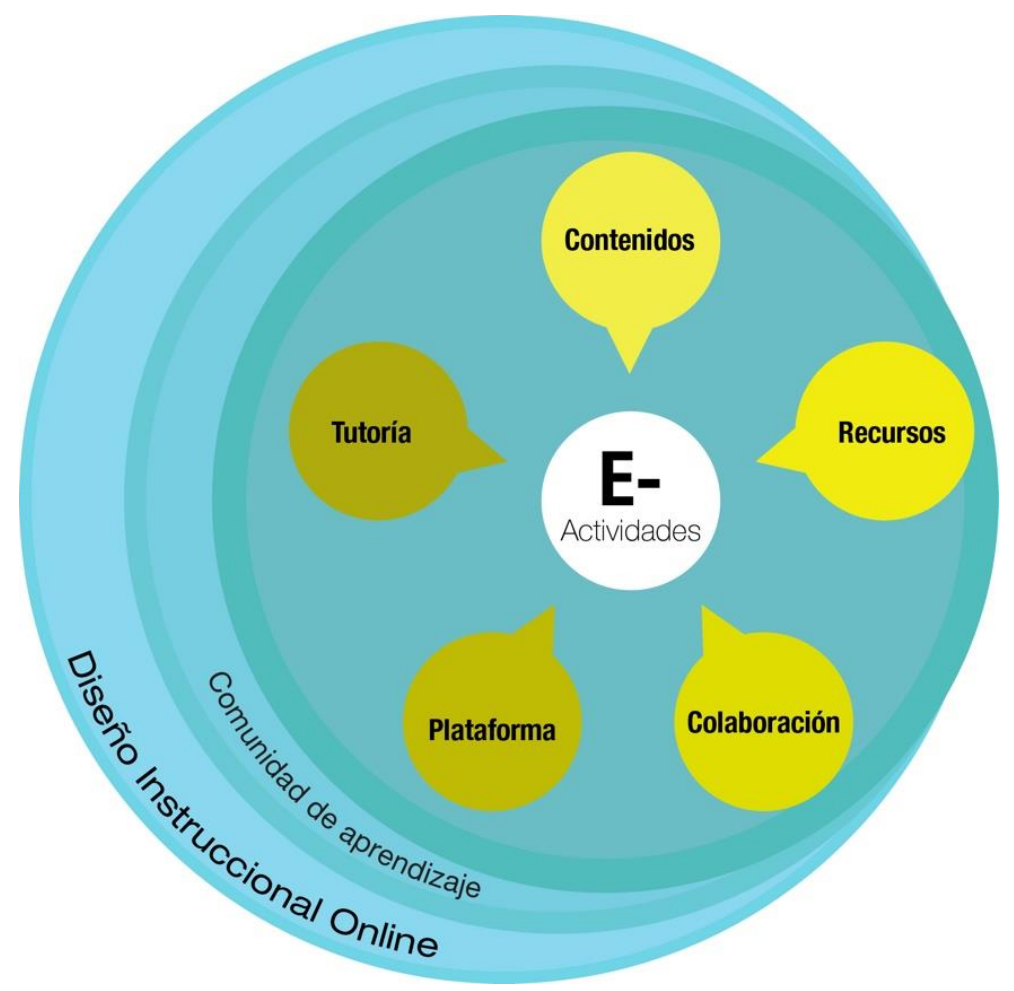

Figura 2: Modelo de EVA centrado en las e-actividades (elaboración propia)

La base del modelo es el diseño instruccional online, que orienta un proceso formativo riguroso y de calidad. Se toman como base el modelo ADDIE (Análisis, Diseño, Desarrollo, Implementación y Evaluación), un proceso de diseño interactivo, donde los resultados de la evaluación formativa de cada fase pueden conducir al regreso a cualquiera de las fases previas, el producto final de una fase es el producto de inicio de la siguiente, se complementa con elementos del modelo ASSURE y el Modelo de Kemp (Muñoz, 2011).

Un segundo aspecto del modelo es la comunidad de aprendizaje, concebida como un espacio donde la interacción entre estudiantes y estudiante tutor son relevantes para la construcción social de conocimiento. Downes (2004), considera que la comunidad de aprendizaje es un entorno educativo superior al del "curso" o "clase reglada". Garrison \& Anderson (2005) proponen centrar la comunidad de aprendizaje en tres elementos constitutivos del e-learning la presencia: social, cognitivo y docente.

El modelo se centra en las e-actividades las cuales apelan a metodologías activas como: búsqueda y procesamiento de la información, proyectos, estudios de casos, juego de roles, la resolución de problemas y el desarrollo colaborativo de productos, discusión. Para definir las actividades se consideran: las características de los participantes, conocimientos previos del curso, nivel de uso de TIC, elementos de la Andragogía. En las e-actividades se despliegan las indicaciones específicas que se deben ejecutar para lograr los objetivos propuestos, lo cuales 
puedes ser conceptuales, actitudinales o procedimentales. Desde las e-actividades se articulan los otros aspectos del curso como contenidos, herramientas de la plataforma, recursos de aprendizaje, evaluación, etc.

La tutoría juega un rol fundamental para acompañar el proceso formativo en sus cuatro dimensiones: administrativa; técnica, pedagógica y social. Los tutores son formados en curso basado en el modelo E-moderating (Salmon, 2000), de esta forma adquieren las habilidades para animar, gestionar y moderar con éxito una comunidad de aprendizaje (Silva \& Astudillo, 2013). Recursos contempla los objetos digitales dispuestos en la plataforma y accesible desde las E-actividades, que buscan favorece el aprendizaje, videos, artículos disponibles en la web, páginas web, simulaciones, etc. Así como las TIC externas a la plataforma contempladas en el desarrollo de las e-actividades como herramientas para generar mapas conceptuales, infografías, lluvias de ideas, blogs, documentos colaborativos, entre otras. Los contenidos, corresponden al material escrito, presentaciones u otros formatos, de autoría del profesor o equipo docente, presentados en herramientas digitales. Cabero (2006) considera que los contenidos son una de las variables críticas en el e-learning y propone mirarlos desde una triple posición: su calidad, su cantidad y su estructuración. La plataforma, considera las herramientas propias del LMS para desplegar contenidos, la colaboración, generación de productos y evaluación. Así como las herramientas para acompañar la tutoría.

\subsection{El modelo en Plataforma}

El modelo toma vida a partir de la estructuración técnica de una plataforma virtual alojada en un LMS, organizada a través de iconos, haciendo posible acceder a este entorno desde diversos dispositivos (PC, tablet o smarthphone). Los iconos permiten al usuario explorar el diseño instruccional en forma libre no hay una ruta definida, el estudiante decide por donde partir y que secuencia seguir, todas las secciones están relacionadas e hipervinculadas. La posibilidad de utilzar iconos en la interfaz, ha permitido contar con interfaces adhoc a la temática del curso.

\subsubsection{Los elementos permanentes}

Elementos permanentes es un espacio transversal al curso, disponible durante toda la ejecución de la experiencia formativa. Los elementos de este espacio obedecen a diferentes propósitos informaciones, motivacionales, sociales y administrativos. 


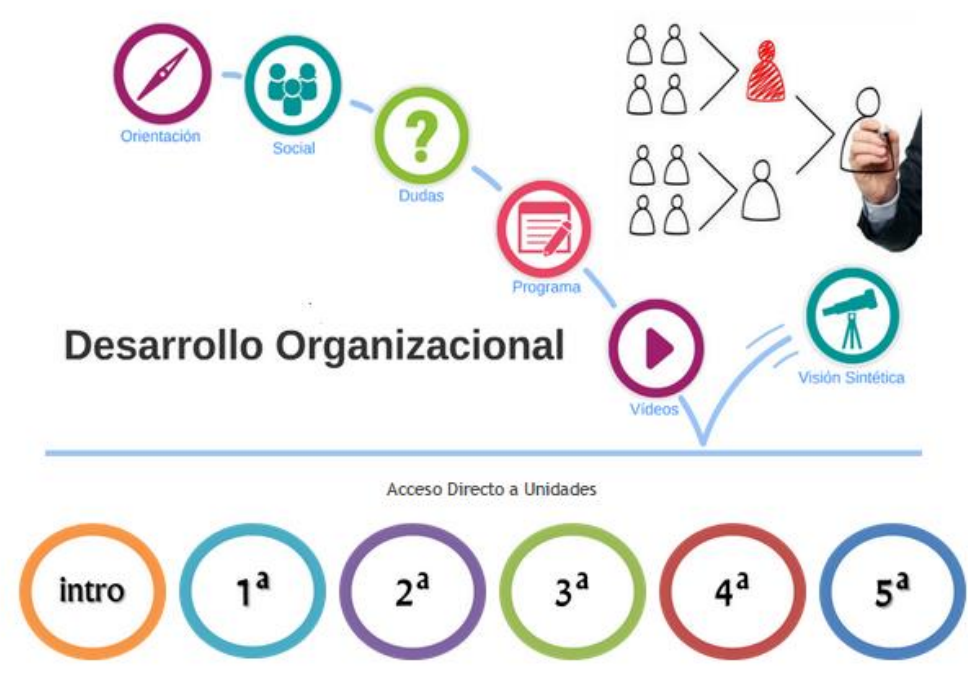

Figura 3: Elementos permanentes (elaboración propia)

Elementos permanentes considera los foros: orientaciones, social y dudas, el programa del curso, un vídeo que presenta el curso protagonizado por el profesor, y la visión sintética que muestra la ruta de aprendizaje. Desde los elementos permanentes se accede a los otros módulos. El estudiante tiene a la vista la interfaz de elementos permanentes y la del módulo en el que está trabajando. Este espacio puede complementarse con otros recursos como: Chat, guiado por el profesor y/o que los estudiantes puedan usar de acuerdo a sus propósitos; Biblioteca general, un espacio que contenga y de acceso a los materiales del curso, podría organizarse por módulo, por tipos de recursos, etc.; Correo electrónico o mensajería interno de la plataforma, para la comunicación personalizada entre los estudiantes y el tutor o entre ellos; Consultas o encuestas, para la recolección rápida de información que permitirá tomar decisiones administrativas o pedagógicas; Cuestionarios para evaluaciones formativas o sumativas.

\subsubsection{Módulo}

El módulo esta conformado por diferentes iconos organizados en forma de red (Díaz \& Ramírez, 2002; Silva 2011, Silva \& Romero, 2014). Este espacio contiene la estructura del módulo, donde las e-actividades son el elemento central. 




Figura 4: Estructura de módulo (elaboración propia)

Presentación: entrega una panorámica, entregando al participante una visión del trabajo a desarrollar en el módulo, sus objetivos, las e-actividades a realizar y el producto esperado asociado a la evaluación. Sitúa al participante en el contexto del módulo.

Contenidos: presenta los contenidos básicos del módulo que el estudiante debe conocer para el trabajo en el módulo. Este material es de autoría del profesor o equipo pedagógico. El contenido es accesible de dos formas: vinculo desde la eactividad, a través de una instrucción que indica su lectura, revisión y/o análisis; en forma directa, en este caso al final se encuentra vínculo a la e-actividad asociada. 


\begin{tabular}{|l|c|} 
Módulo 1 & Contenidos \\
\hline
\end{tabular}

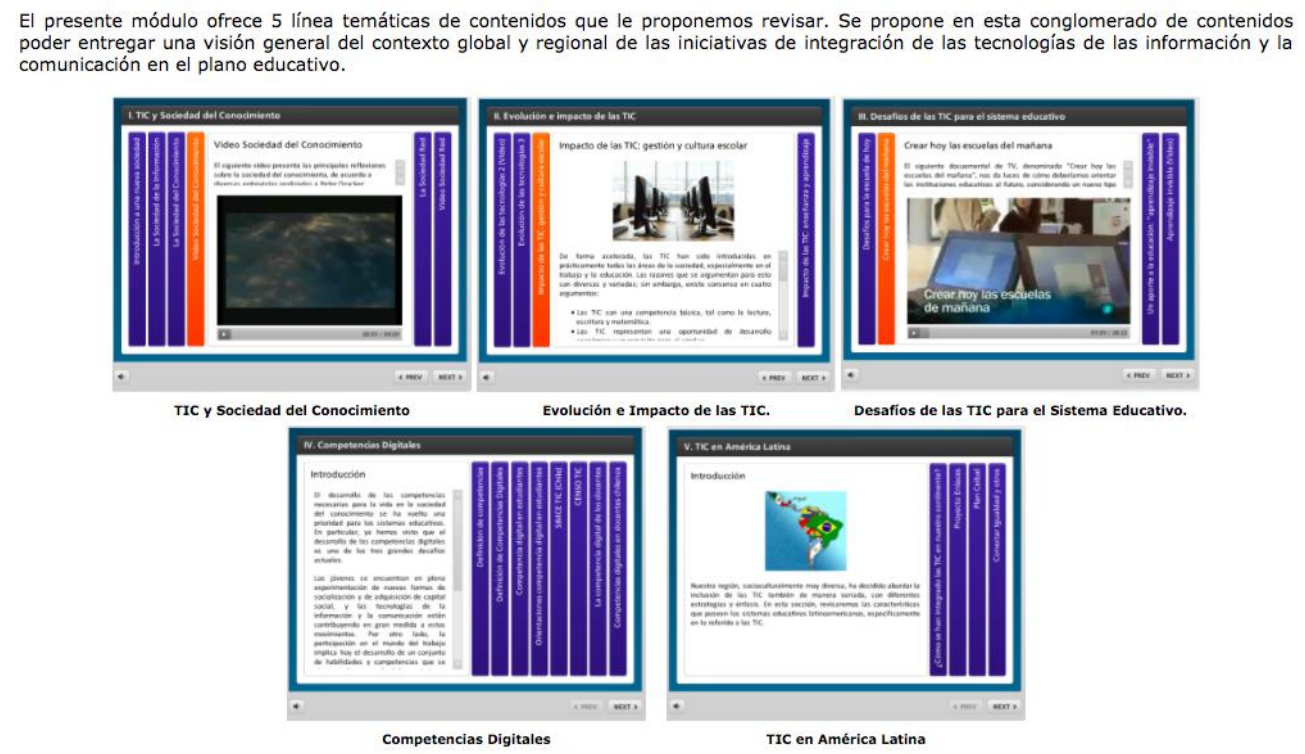

Figura 5: Contenido de módulo (elaboración propia)

Evaluación: presenta la evaluación asociada a cada actividad y al producto del módulo. Las actividades sumativas y producto final, consideran instrumentos de evaluación, como pautas de cotejos, rubricas u otras. Las actividades formativas contienen instrumentos para la autoevaluación.

Biblioteca: presenta los diferentes recursos obligatorios y complementarios contemplados en las e-actividades como: documentos, presentaciones, videos, páginas web, etc. los obligatorios como complementarios. Permite acceder a los recursos en forma directa, fuera del contexto de la e-actividad.

Herramientas: provee acceso directo a los recursos de la plataforma utilizados en las e-actividades, para compartir e interactúa con los compañeros y el tutor, construcción personal o colaborativa, enviar productos tareas y para la evaluación.

E-Actividades: es elemento central del diseño instruccional y a través de una estructura flexible despliega la actividad, considera los siguientes aspectos: Titulo, Descripción, Objetivos, Competencia o Resultados de Aprendizaje, Tiempo, Actividades de apropiación, Evaluación y Actividades Complementarias. Las Actividades de apropiación son el conjunto de acciones obligatorias para el logro de los objetivos propuestos, desde ellas se vincula al conjunto de materiales y recursos internos y externos; La Evaluación señala la forma en que se evaluará la actividad; las Actividades complementarias permiten profundizar en las temáticas de la e-actividad. 
Como se pudo apreciar en el módulo 0 , introductorio a la plataformar, es necesario realizar algunas actividades que afianzan el proceso de aprendizaje realizado hasta ahora:

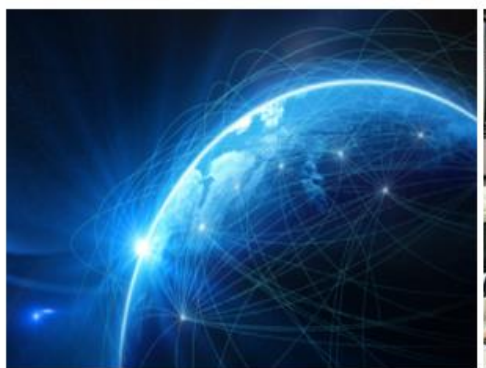

Actividad 1: Reflexionando sobre la nueva sociedad.

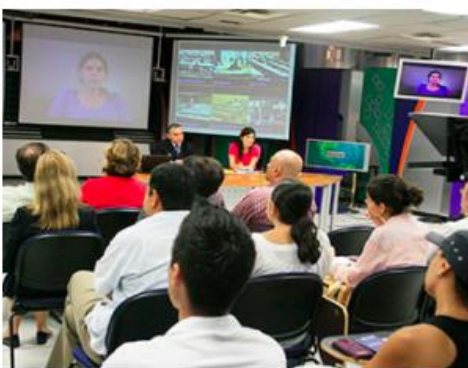

Actividad 2: Impacto de las TIC sobre mi escuela.

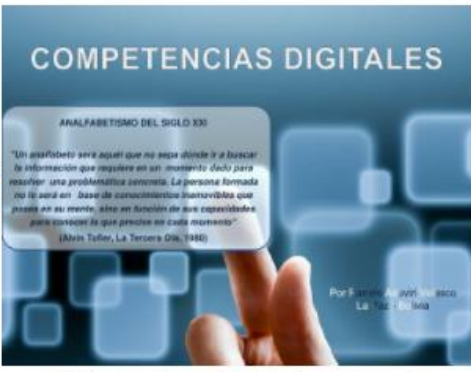

Actividad 3: Autoevaluación de competencias
digitales.

Figura 6: Presentación de las actividades del módulo (elaboración propia)

La actividad se despliega en la estructura descrita anteriormente, en una página web

La presente actividad tiene por finalidad substraer conceptos elementales asociados a los procesos de formación a distancia con el uso de tecnologias de la información y la comunicación (TIC). objetivos:

- Caracterizar la Eđucación a Distancia mediada por las tecnologías de la información y comunicación

- Diferencias las principales modalidades de la formación a distancia mediadas por las tecnologías de la información y la comunicación. Duración: 8 horas.

Actividad de Apropiación:

1. Le sugerimos revisar el contenido №2: Formación a Distancia TIC y el siguiente vídeo sobre el futuro de la Formación a Distancia:



2. De acuerdo al contenido revisado, extraiga a lo menos 6 conceptos referidos a la FAD mediada por TIC, busque el significado de los conceptos y comparta su definición en Glosario: Conceptos de FAD mediados por TIC. (antes de publicar los conceptos revise que no se encuentren en el glosario). Para esto tome en cuenta lo siguiente:

- Nombre del concepto. (nombre completo y/o sigla)

- Definición del concepto.

Fuen alusiva. (no es obligatorio)

3. Revise los conceptos publicados por sus compañeros y comente a lo menos 3 de ellos, complementando el concepto 0 enriqueciendolo con sus observaciones.

Evaluación:

Los criterios de evaluación asociados al producto de esta actividad son los siguientes:

- Los conceptos son los adecuados. Presenta más de 6 conceptos.

Figura 7: Actividad de módulo (elaboración propia) 
Las e-actividades contemplan diversas acciones que utilicen diferentes recursos de la web 2.0 como: construcción colaborativa de documentos, mapas conceptuales, elaboración de presentaciones, generación de infografías, diseño de vídeos, etc. Además de herramientas de la plataforma como: base de datos, glosario, wiki, coevaluación, foros, tareas, etc.

\section{Evaluación del Modelo}

Para evaluar el modelo dentro de una encuesta general de satisfacción de los cursos que dicta CIIET en modalidad virtual, esto es, materiales, contenidos, objetivos, tutoría, entre otros, se incorporaron preguntas para medir diferentes aspectos del modelo. La encuesta fue respondida por 320 participantes, 85 corresponden a un curso de formación por competencias, y 235 a un del curso de formación de tutores online. La encuesta se diseño y aplicó en Google Formulario. En ambos casos los participantes son profesionales que desempeñan un rol docente en las instituciones de las cuales provienen.

La interface gráfica que presenta la plataforma para navegar en los distintos espacios de los módulos de aprendizaje, alcanza una evaluación promedio de 6.2 (1: muy mala / 7:muy buena), un $89 \%$ del puntaje máximo. Respecto a la utilidad de la conectividad que presente la plataforma entre sus diferentes componentes, actividades, contenidos, etc, el puntaje promedio alcanza a 6.0 (1:poco útil / 7:muy útil), un $86 \%$ del máximo

La pertinencia de la propuesta metodológica general del curso, en lo que respecta a sus actividades y procesos de evaluación, relacionados con los objetivos del programa de formación, presenta una valoración promedio es 6.3 (1: No pertinente / 7: Muy pertinente), un 90\% del máximo. En relación a centrar el aprendizaje en el estudiante, al ser éstos consultados si el programa de formación que ha realizado, esta basado en un enfoque que busca el desarrollo de acciones pedagógicas o actividades centradas en el estudiante, para la construcción de conocimiento a partir de productos o evidencias de aprendizaje, consultados si este enfoque satisface sus necesidad de aprendizaje, se alcanza un puntaje promedio de 6.4 (1: Insatisfactorio / 7: Muy satisfactorio) un 91\% del puntaje máximo. La evaluación a partir de la observación verificable de productos o evidencias de aprendizaje, por sobre otros instrumentos como pruebas o test, presenta un puntaje promedio de 6.3 (1: Insatisfactorio / 7: Muy satisfactorio) un 90\% del puntaje máximo.

El centro del modelo son las e-actividades, para medir este aspecto y su relación con los contenidos, materiales y evaluación, se realizaron una serie de preguntas (tabla 2) que los participantes valoraron en una escala de liker de 1 a 4 , donde 1 corresponde a totalmente en desacuerdo y 4 totalmente de acuerdo. 


\begin{tabular}{|l|c|c|c|c|}
\hline & $\begin{array}{l}\text { Totalmente en } \\
\text { Desacuerdo }\end{array}$ & Desacuerdo & Acuerdo & $\begin{array}{c}\text { Totalmente } \\
\text { de Acuerdo }\end{array}$ \\
\hline Los contenidos fueron pertinentes & $2 \%$ & $2 \%$ & $32 \%$ & $64 \%$ \\
\hline $\begin{array}{l}\text { Las actividades fueron } \\
\text { consistentes con los contenidos } \\
\text { del curso }\end{array}$ & $2 \%$ & $2 \%$ & $29 \%$ & $67 \%$ \\
\hline $\begin{array}{l}\text { Las actividades son útiles para el } \\
\text { desempeño profesional }\end{array}$ & $2 \%$ & $2 \%$ & $23 \%$ & $73 \%$ \\
\hline $\begin{array}{l}\text { Las actividades cumplen su } \\
\text { propósito y facilitan el logro de } \\
\text { los objetivos del curso }\end{array}$ & $2 \%$ & $3 \%$ & $31 \%$ & $65 \%$ \\
\hline $\begin{array}{l}\text { Las actividades fueron } \\
\text { pertinentes para el aprendizaje de } \\
\text { los contenidos }\end{array}$ & $2 \%$ & $4 \%$ & $28 \%$ & $66 \%$ \\
\hline $\begin{array}{l}\text { Existe coherencia entre las } \\
\text { actividades y los contenidos del } \\
\text { curso }\end{array}$ & $3 \%$ & $8 \%$ & $39 \%$ & $50 \%$ \\
\hline $\begin{array}{l}\text { Las actividades fomentan el } \\
\text { trabajo colaborativo }\end{array}$ & $3 \%$ & $8 \%$ & $34 \%$ & $55 \%$ \\
\hline $\begin{array}{l}\text { Los medios, materiales y recursos } \\
\text { didácticos presentes son } \\
\text { facilitadores para el aprendizaje. }\end{array}$ & $2 \%$ & $5 \%$ & $33 \%$ & $59 \%$ \\
\hline $\begin{array}{l}\text { Existe coherencia entre la } \\
\text { evaluación, objetivos, contenidos } \\
\text { y estrategias utilizadas. }\end{array}$ & $3 \%$ & $35 \%$ & $59 \%$ \\
\hline
\end{tabular}

Tabla 2: Evaluación de las E-actividades

Existe en general una positiva valoración de los diferentes indicadores todos están sobre el $89 \%$ de una valoración acuerdo o total acuerdo. Los indicadores respecto a si las actividades fueron consistentes con los contenidos y si éstas son útiles para el desempeño profesional alcanzan un $96 \%$ de acuerdo o total acuerdo. El indicador menor evaluado es existe coherencia entre las actividades y contenidos del curso, alcanza un $89 \%$ de valoración acuerdo y total acuerdo.

\section{Conclusiones}

Los sistemas formativos requieren adaptar sus metodologías a las necesidades de la sociedad actual y las demandas de los propios estudiantes. En este contexto las TIC aparecen como una agente de transformación, siendo las plataformas virtuales el estandarte de este cambio, sin embargo la incorporación de un EVA, no garantiza la innovación ni la mejora de la calidad de la enseñanza, es necesario modificar los modelos pedagógicos, colocar al estudiante al centro del proceso, lo que implica ubicar las e-actividades al centro del diseño pedagógico e incorporar 
metodologías activas y aprovechar el conectivismo que nos ofrecen las herramientas TIC.

Diseñar e implementar un EVA centrado en el estudiante, requiere ubicar desde el diseño instruccional online las e-actividades en el centro del proceso formativo en lugar de los contenidos. No implica que éstos desaparezcan sino que están al servicio de la actividad. Esta apuesta formativa demanda cambios en el rol del docente y alumno. Es un ejercicio que tiene sus complejidades, el profesor está acostumbrado a pensar en el contenido no en la actividad, ésta es complementaria. Lograr este cambio en el diseño, transforma la práctica docente, independiente de la modalidad en que se enseñe.

Las e-actividades son el detonador que permita motivar a la comunidad de aprendizaje a compartir, construir y colaborar, desarrollando el aprendizaje social y enriqueciendo el autoaprendizaje. Un EVA centrado en las actividades es una innovación educativa que permite una mayor sintonía con la sociedad actual, que busca que los estudiantes desarrollen competencias de orden superior como el trabajo en equipo, autonomía y colaboración.

Fecha de presentación del artículo: 15 de Noviembre de 2016

Fecha de aprobación: 15 de Marzo de 2017

Fecha de publicación: 31 de Marzo de 2017

Silva, J. (2017). Un modelo pedagógico virtual centrado en las E-actividades. RED. Revista de Educación a Distancia, 53. Consultado el (dd/mm/aaaa) en http://www.um.es/ead/red/53/silva.pdf

\section{Referencias}

Adell, J. Castellet J y Pascual, J. (2004). Selección de un entorno virtual de enseñanza/aprendizaje de código fuente abierto para la Universitat Jaume. Recuperdo de http://cent.uji.es/doc/eveauji_es.pdf

Adell, J. (2004). Nuevas tecnologías en la formación presencial: del curso on-line a las comunidades de aprendizaje, Qurriculum: Revista de teoría, investigación y práctica educativa, 17, 57-92. Recuperado de http://elbonia.cent.uji.es/jordi/wp-content/uploads/docs/Qurriculum_2004.pdf

Barberà, E. \& Badia, A. (2004) Educar con aulas virtuales: Orientaciones para la innovación en el proceso de enseñanza y aprendizaje, Madrid: Machado.

Bolívar, J. \& Rojas, F. (2014). Estudio de la autopercepción y los estilos de aprendizaje como factores asociados al rendimiento académico en estudiantes universitarios. RED, Revista de Educación a Distancia. Número 44. Número monográfico sobre "Buenas prácticas de Innovación Educativa: Artículos seleccionados del II Congreso Internacional sobre Aprendizaje, Innovación y Competitividad, CINAIC 2013”. Recuperado de http://www.um.es/ead/red/44 
Bullen, M. \& Morgan, T. (2015). Digital Learners in Higher Education: Implications for Teaching, Learning \& Technology. In M. Gisbert \& M. Bullen (Eds.). Teaching and Learning in Digital Worlds: Strategies and Issues in Higher Education, 11-19. Tarragona, Spain

Cabero, J. (2006). Bases pedagógicas del e-learning. RUSC Revista de Universidad y Sociedad del Conocimiento Journal, 3 (1). Recuperado de www.uoc.edu/rusc/3/1/dt/esp/cabero.pdf

Cabero, J. y Román, P. (2006). E-actividades. Un referente básico para la formación en Internet. Sevilla: Eduforma.

Carrasco, R., Jadue, F., Letelier, M. \& Oliva, C. (2012). Estudio exploratorio sobre aprendizaje no formal e informal de estudiantes y egresados universitarios. Calidad de la Educación, 1er semestre (36), 150-183.

Corno, J. \& Randi, L. (2000). ¿Una teoría del diseño educativo basado en el aprendizaje autorregulado?, en Reigeluth, C. (coord.) Diseño de la instrucción: teorías y modelos: un nuevo paradigma de la teoría de la instrucción.Vol1. Madrid: Santillana; 305-334.

Diaz J. \& Ramirez T (2002). Un Modelo de diseño instruccional para la elaboración de cursos en línea. Universidad Veracruzana Virtual. Recuperado de

http://www.uv.mx/jdiaz/DisenoInstrucc/ModeloDisenoInstruccional2.htm\#

Dillenbourg, P (2000). Virtual learning environments, Workshop on virtual learning. Recuperado de http://tecfa.unige.ch/tecfa/publicat/dil-papers-2

Downes, S. (2004). Educational Blogging. EDUCAUSE Review, 39(5), 14-26. Recuperado de www.educause.edu/pub/er/erm04/erm0450.asp.

Duart, J,, Gil. M., Pujol, M \& Castaño, J. (2008), La Universidad en la sociedad RED, usos de la internet en educación superior, Barcelona: Arie

Esteve, F., Duch, J. \& Gisbert, M. (2014). Los aprendices digitales en la literatura científica: diseño y aplicación de una revisión sistemática entre 2001 y 2010. Pixel-Bit, Revista de Medios y Educación, 45, 9-21. http://dx.doi.org/10.12795/pixelbit.2014.i45.01

García Aretio, L. (coord.), Ruiz, M. \& Domínguez, D. (2007). De la educación a distancia a la educación virtual, Ariel: Barcelona.

García. I. Gros, B. \& Escofet, A. (2012), La influencia del género en la cultura digital del estudiantado universitario, Athenea Digital: revista de pensamiento e investigación social, 12(3), 95-114. .

http://dx.doi.org/10.5565/rev/athenead/v12n3.1075

Garrison, D.R \& Anderson, T. (2005), El e-learning en el siglo xxi: Investigación y práctica, Barcelona : Octaedro 
Gisbert, M. \& Johnson, L. (2015). Educación y tecnología: nuevos escenarios de aprendizaje desde una visión transformadora. Universities and Knowledge $\begin{array}{lllll}\text { Society Journal (RUSC). } 12 & \text { (2). págs. 14. }\end{array}$ http://dx.doi.org/10.7238/rusc.v12i2.2570

Gisbert, M., \& Lázaro, J. L. (2015). Professional development in teacher digital competence and improving school quality from the teachers' perspective: a case study. NAER: New Approaches in Educational Research, 4(2), 115122. http://dx.doi.org/10.7821/naer.2015.7.123

Gros (2004) La construcción del conocimiento en la red: límites y posibilidades. Revista Teoría de la Educación: Educación y Cultura en la Sociedad de la Información, Vol. $\quad 5 . \quad$ Recuperado de http://www3.usal.es/ teoriaeducacion/rev_numero_05/n5_art_gros.htm

Gros, B. (2011). Evolución y retos de la educación virtual: construyendo en el siglo xxi. Barcelona: Editorial UOC.

Honey, P. \& Mumford, A. (2000).The learning styles helper's guide. Maidenhead: Peter Honey Publications Ltd.

Kennedy, G., Judd, T. S., Churchward, A., Gray, K., \& Krause, K.-L. (2009). First year stu- dents' experiences with technology: Are they really digital natives? Australasian Journal of Educational Technology, 24(1), 108-122

Oblinger, D. G., \& J. L. Oblinger, (2005). Educating the Net Generation. Washington, D.C.: EDUCAUSE

Pedró, F. (2006). Aprender en el nuevo milenio: Un desafío a nuestra visión de las tecnologías y la enseñanza. OECD-CERI.

Labrador, M, y Andreu, M. (2008). Metodologías activas. Valencia: Universidad Politécnica de Valencia.

Mason, R. (1998, octubre). Models of online courses. ALN Magazine, 2(2).

Martín, M. \& López, E. (2012) La Sociedad de la Información y la Formación del Profesorado. E-actividades y Aprendizaje. RIED. Revista Iberoamericana de Educación a Distancia. $15 \quad$ (1). 15-35. Recuperado de http://revistas.uned.es/index.php/ried/article/view/775/686

Miranda, M. J., Guerra, L., Fabbri, M. \& López, E. (2010). Experiencias universitarias de innovación docente hispano-italianas en el espacio europeo de educación superior. Sevilla: Mergablum.

Muñoz, P (2011). Modelos de diseño instruccional utilizados en ambientes teleformativos. Revista Digital de Investigación Educativa Conect@2, 2(1). Recuperado de http://www.revistaconecta2.com.mx/archivos/revistas/revista2/2_2.pdf 
Roig, R., Rioseco, M. y Belmar, M.(2015). Expectativas de estudiantes universitarios frente a sus capacidades y competencias para participar en cursos abiertos y en línea. RED. Revista de Educación a Distancia. 47(2). http://www.um.es/ead/red/47/Roig_et_al.pdf

Salinas, J. (2004). Innovación docente y uso de las TIC en la enseñanza universitaria. RUSC Revista de Universidad y Sociedad del Conocimiento Journal, 1(1), http://dx.doi.org/10.7238/rusc.v1i1.228

Salinas, J., Pérez, A. \& De Benito, B. (2008). Metodologías centradas en el alumno para el aprendizaje en red, Madrid: Síntesis.

Salmon, G. (2000). E-moderating: The key to teaching and learning online, London: Kogan Page.

Salmon, G. (2004). E-Actividades, El factor clave para una formación en línea activa, Barcelona: Editorial UOC.

Silva, J. (2011). Diseño y moderación de Entornos Virtuales de Aprendizaje, Barcelona: Editorial UOC.

Silva, J. \& Romero, M. (2014). La virtualidad una oportunidad para innovar en educación: Un modelo para el diseño de entornos virtuales de aprendizaje Didasc@lia: Didácticay Educación. 5 (1), 1-22.

Villalustre, L. \& María Del Moral (2011) . E-actividades en el contexto virtual de Ruralnet: Satisfacción de los estudiante con diferentes estilos de aprendizaje. Educación XXI. 14(1), 223-243. http://dx.doi.org/10.5944/educxx1.14.1.270 